\title{
Optical-geometrical effects on the photoluminescence spectra of Si nanocrystals embedded in $\mathrm{SiO}_{2}$
}

\author{
R. Ferre, B. Garrido, ${ }^{\text {a) }}$ P. Pellegrino, ${ }^{\text {b) }}$ M. Perálvarez, C. García, J. A. Moreno, \\ J. Carreras, and J. R. Morante \\ Electronic Materials and Engineering (EME), Department d'Electrònica, Universitat de Barcelona, \\ Martí i Franquès 1, 08028 Barcelona, Spain
}

(Received 13 April 2005; accepted 13 September 2005; published online 31 October 2005)

\begin{abstract}
We demonstrate that thickness, optical constants, and details of the multilayer stack, together with the detection setting, strongly influence the photoluminescence spectra of Si nanocrystals embedded in $\mathrm{SiO}_{2}$. Due to multiple reflections of the visible light against the opaque silicon substrate, an interference pattern is built inside the oxide layer, which is responsible for the modifications in the measured spectra. This interference effect is complicated by the depth dependence of (i) the intensity of the excitation laser and (ii) the concentration of the emitting nanocrystals. These variations can give rise to apparent features in the recorded spectra, such as peak shifts, satellite shoulders, and even splittings, which can be mistaken as intrinsic material features. Thus, they can give rise to an erroneous attribution of optical bands or estimate of the average particle size, while they are only optical-geometrical artifacts. We have analyzed these effects as a function of material composition ( $\mathrm{Si}$ excess fraction) and thickness, and also evaluated how the geometry of the detection setup affects the measurements. To correct the experimental photoluminescence spectra and extract the true spectral shape of the emission from Si nanocrystals, we have developed an algorithm based on a modulation function, which depends on both the multilayer sequence and the experimental configuration. This procedure can be easily extended to other heterogeneous systems. (C) 2005 American Institute of Physics. [DOI: 10.1063/1.2115100]
\end{abstract}

\section{INTRODUCTION}

Si nanocrystals (Si-ncs) in a silicon oxide matrix are attractive for optoelectronics and photonics as a consequence of their visible and tunable photoluminescence (PL) and electroluminescence (EL), optical gain, and nonlinear optical properties. ${ }^{1-4}$ The emission bands of these $\mathrm{Si}-\mathrm{SiO}_{2}$ composites have been studied as a function of silicon excess for materials obtained from different fabrication methods. ${ }^{5,6}$ The emission band coming from Si nanocrystals embedded in $\mathrm{SiO}_{2}$ appears after $\mathrm{Si}$ excess precipitation into nanocrystals during a high-temperature annealing and it peaks between 1.4 and $1.7 \mathrm{eV}$. During nucleation and growth of the Si-ncs, their emission intensity increases with annealing time, until a saturation regime is reached when most of the Si excess has precipitated and interfacial defects have been annealed out. ${ }^{7}$

Several authors have reported shifts of the emission peaks, appearance of satellite bands, and even the splitting of the main emission band as a consequence of material variations such as nanocrystal size and density. ${ }^{1,5-12}$ Shifting shoulders around the main band have been attributed to intrinsic properties such as the existence of optically active defects. ${ }^{12}$ Together with specific structural properties, these PL spectra modifications have been used to tentatively validate the mechanisms of emission based on quantum confinement, interfacial radiative defects, or others. The correlation of the PL spectra with material structure and emission mechanism which has been provided by many authors is

\footnotetext{
a)Electronic mail: blas@el.ub.es

${ }^{b)}$ Electronic mail: paolo@el.ub.es
}

usually self-consistent, especially when they compare samples with similar thickness and fabricated in the same experimental conditions. ${ }^{6-12}$ However, comparisons between the results obtained by different groups are always difficult because experimental values of peak energies, widths, and intensities of spectral bands are scattered over a wide range. Moreover, several research groups have tentatively tried to reproduce the observed position and shape of the PL band from the measured spread in size distribution. For this purpose they made use of theoretical calculations of the variation of the electronic band gap of the nanocrystalline silicon with the particle size, based on the quantum confinement model. ${ }^{13-15}$

On the other hand, only scarce information is available in the literature about how the PL spectra can be dramatically influenced by optical-geometrical features such as layer thickness, refractive index, multilayer sequence and geometry of the excitation, and collection setups. These effects arise as a consequence of the optical response of the layered system, considering for the interference patterns both the profile of the excitation radiation and the depth distribution of the emitting Si-ncs. Oscillation patterns in the emission spectra of $\mathrm{Si}-\mathrm{SiO}_{2}$ samples grown on a $\mathrm{Si}$ substrate and their absence in identical samples formed on a silica substrate have been mentioned by Iwayama et al. ${ }^{1,5}$ However, they do not provide with a dedicated study of this phenomenon. Nesheva et al. have pointed out that oxides thinner than $0.2 \mu \mathrm{m}$ must be chosen to avoid interference effects. ${ }^{16}$ Orbons et al. have reported the distortion of the PL spectra as a function of sample thickness only for a single $\mathrm{Si}$ ion implantation in 
silica. ${ }^{17}$ Elliman et al. have highlighted how such interferences can affect the transmittance and reflectance spectra also in very thick silica samples containing Si-ncs, due to their modification of the refractive index of the material. ${ }^{18}$

In this paper our experimental approach extends the analysis of the optical response to samples with a planar profile of $\mathrm{Si}$ excess (well-defined refractive index contrast and much simpler structure) and to a series of different $\mathrm{Si}$ excesses (variation in refractive index), allowing us to determine in a straightforward and reliable way the impact of material structure on the PL spectra. We report as well the experimental results and modeling performed in order to (i) demonstrate how the recorded PL spectra can be affected by optical interference, (ii) quantify its magnitude, and (iii) propose a procedure to eliminate/minimize its effects. We have developed a simulation tool, which allows modeling of the spectra in Si-rich oxides grown on a $\mathrm{Si}$ substrate by using a modulation function that accounts for both the optical response of the multilayer stack and the detection setup geometry.

\section{EXPERIMENT}

A 300-nm-thick buried layer with uniform Si excess in $\mathrm{SiO}_{2}$ has been obtained by performing $\mathrm{Si}^{+}$multi-implantation into two different substrates: 2-mm-thick fused silica (FS) glass or $2-\mu$ m-thick $\mathrm{SiO}_{2}$ thermally grown on $\mathrm{Si}$ thermal oxide (TO) wafers. TRIM simulations have provided the correct doses and energies of implantation. Three samples for each type of substrate have been synthesized with different Si excesses (from 7\% up to 13\%). The phase separation and precipitation of Si-ncs have been attained by annealing the samples at $1100{ }^{\circ} \mathrm{C}$ for $4 \mathrm{~h}$. As we have previously demonstrated, this treatment ensures the precipitation of most of the Si excesses. ${ }^{7}$ X-ray photoelectron spectroscopy (XPS) measurements have been carried out to estimate the actual $\mathrm{Si}$ excess and its in-depth profile. Transmission electron microscopy (TEM) has been used to image the Si-ncs and check the uniformity of the size distribution.

Photoluminescence measurements have been performed by using for excitation a $30 \mathrm{~mW} \mathrm{He}-\mathrm{Cd}$ laser emitting at 325 $\mathrm{nm}$. In order to vary the incident and collection angles, the sample has been positioned onto a goniometer placed in the focus of a spherical mirror, which collects the light emitted from the sample and focuses it at the entrance of a $0.5 \mathrm{~m}$ monochromator. A GaAs photomultiplier tube has been used as a detector and standard chopper/lock-in synchronous detection has been applied to minimize the noise. Great care has been taken in measuring and correcting the spectral response of the whole detection system. Figure 1 shows the schematics of the experimental setup. One of the critical parameters to control is the detection angle $\varepsilon$ (see Fig. 1 for details). Since the spherical mirror used for collecting the emitted light has an aperture of about $16^{\circ}$, which sets the angle resolution of the system, the value of $\varepsilon$ has to be taken as an averaged value. The influence of this aperture on the experimental spectra has been checked out and consists of a small increase in the width of the emission band, while the position of the maximum does not show any variation.

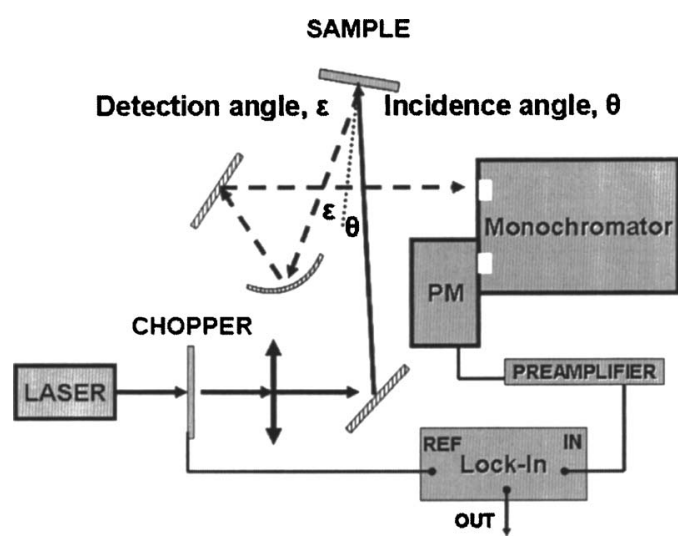

FIG. 1. Schematics of the photoluminescence experimental setup with incident and detection geometries indicated.

\section{PHYSICAL AND OPTICAL CHARACTERIZATIONS}

Figure 2 shows a typical XPS profile which can be briefly described as a central flat region with thickness $d_{g}$ $=300 \mathrm{~nm}$ of Si excess starting at a depth $d_{0}$ of about $50 \mathrm{~nm}$. For each implanted dose the actual $\mathrm{Si}$ excesses measured are $7 \%, 9.5 \%$, and $13 \%$, respectively. A dark-field TEM picture of the sample with $13 \%$ of $\mathrm{Si}$ excess is shown in Fig. 3. Si nanocrystal size varies between $3.6 \mathrm{~nm}$ for the $7 \% \mathrm{Si}$ excess and $4.6 \mathrm{~nm}$ for the $13 \% \mathrm{Si}$ excess, as indicated in Table I. Within the $300 \mathrm{~nm}$ region of the flat profile, size distribution is independent of depth. By assuming a perfect phase separation and by combining Si excess and size measurements, we have estimated a density of about $4 \times 10^{18} \mathrm{Si}-\mathrm{nc} / \mathrm{cm}^{3}$, which appears roughly independent of the $\mathrm{Si}$ excess. All these data will be used in the following to perform the simulations. Exhaustive details of these measurements and additional secondary-ion-mass spectroscopy (SIMS) and Rutherford backscattering spectroscopy (RBS) experiments on the same samples are provided elsewhere. ${ }^{19}$

Figure 4 shows the modification of the PL spectrum when varying the $\mathrm{Si}$ excess in thermal oxide and fused silica samples, respectively, for a quasinormal incidence/detection setting. The intensities have been normalized to magnify the shift as a function of Si excess. Even if the following discussion will be focused mainly just on the spectral features, in general a stronger emission has been observed from the

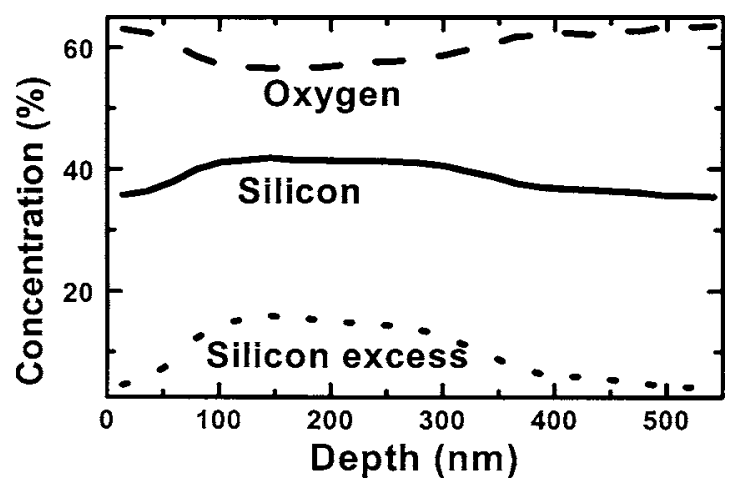

FIG. 2. Concentration profiles vs depth for a fused silica sample implanted with $13 \% \mathrm{Si}$ excess, as obtained from x-ray photoelectron spectroscopy measurements. The silicon excess has been calculated from the measured oxygen and silicon relative concentrations. 


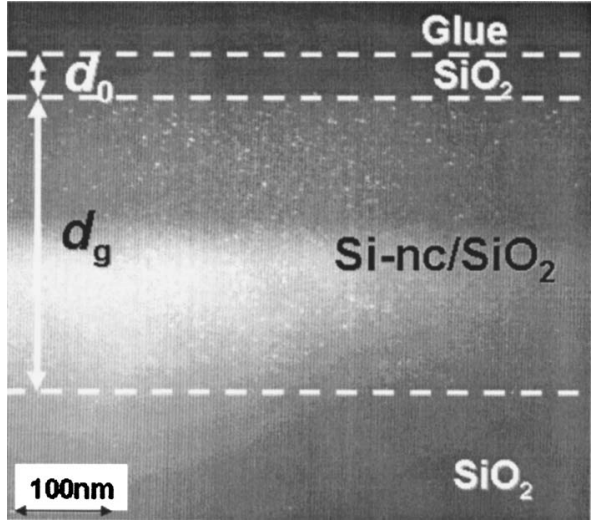

FIG. 3. Image of the active layer with a $13 \%$ silicon excess from transmission electron microscopy in dark field.

samples grown on a silicon substrate. The Si substrate reflects backwards the weakly absorbed excitation radiation, enhancing the power density available for the excitation of the nanocrystal ensemble and resulting in a PL emission efficiency up to the double of the corresponding layer buried in fused silica. In Fig. 4 it is apparent that for increasing $\mathrm{Si}$ excess, i.e., Si grain size, the peak energy position of the FS spectra redshifts, in agreement with the quantum confinement theory. It is worth to stress that these spectra show no distortion coming from interference effects in the layers. The redshifts are much more reduced in the PL spectra of the corresponding TO samples, which show distortions and a pronounced shoulder in the high-energy side of the spectra, for this particular chosen experimental configuration. Moreover, contrary to the TO spectra, emission bands from FS samples can almost perfectly be fitted by a Gaussian shape $250 \mathrm{~nm}$ width, with an accuracy larger than $99.5 \%$. In fact, FS wafers can be considered as semi-infinite media, as the interference pattern in the 2-mm-thick wafer will roughly give maxima separated by $\lambda_{2}-\lambda_{1}$, where

$$
2 n_{0} d \cos \theta=\left(1 / \lambda_{2}-1 / \lambda_{1}\right)^{-1},
$$

and result in $0.1 \mathrm{~nm}$, considering $n_{0}=1.46$ the refractive index of $\mathrm{SiO}_{2}, d$ the total thickness of the $\mathrm{SiO}_{2}$ wafer, and $\theta$ $=0^{\circ}$ as the incidence angle. Thus no modulation can be observed with the resolution usually available, and this will be true for very thick layers, usually substrates. Hence, the spectra from FS samples can be regarded as the actual spectra from Si-ncs containing no distortion. On the contrary, emis-

TABLE I. Implanted Si excess and corresponding nanocrystal density and size.

\begin{tabular}{cccc}
\hline \hline & \multicolumn{2}{c}{$\begin{array}{c}\text { Si nanocrystals } \\
\text { size }\end{array}$} & \\
\cline { 2 - 3 } $\begin{array}{c}\text { Si excess in the } \\
\text { implanted } \\
\text { layer (at. \%) }\end{array}$ & $\begin{array}{c}\text { Mean } \\
\text { value } \\
(\mathrm{nm})\end{array}$ & $\begin{array}{c}\text { Dispersion } \\
(\mathrm{nm})\end{array}$ & $\begin{array}{c}\text { Density of Si } \\
\text { nanocrystals } \\
\left(\times 10^{18} \mathrm{~cm}^{-3}\right)\end{array}$ \\
\hline 13 & 4.6 & 0.6 & 4 \\
9.5 & 4.1 & 0.5 & 4 \\
7 & 3.6 & 0.4 & 4 \\
\hline \hline
\end{tabular}

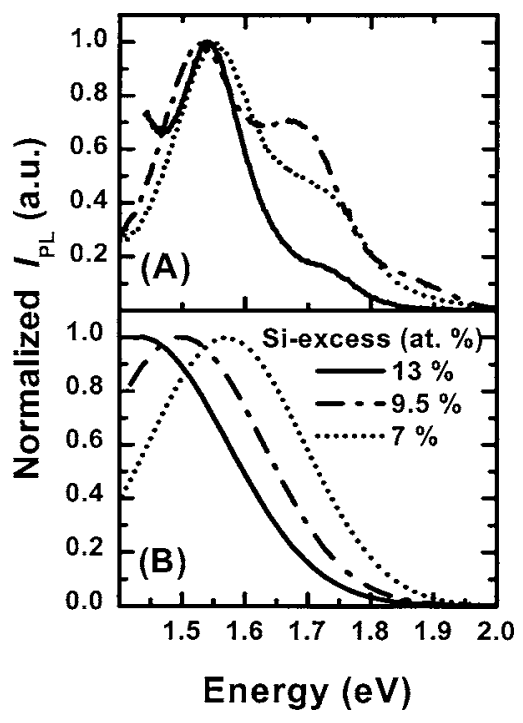

FIG. 4. Normalized photoluminescence intensity spectra for different silicon contents in thermal oxide (a) and fused silica (b) samples.

sion shapes coming from the $2 \mu \mathrm{m}$ TO samples are much more complicated, as the energy scale of the modulation within the thermal oxide layer will be roughly $244 \mathrm{meV}$, a value comparable to the width of the emission band. Thermal oxides with a thickness less than $0.3 \mu \mathrm{m}$ would not show significant interference effects as the semiperiod of the modulations would cover more than the whole visible spectrum. Then, we can conclude that, despite both the FS and TO samples have undergone the same processing steps and present the same Si-nc in-depth structure, they display significant differences in peak position, width, and shape of the PL bands. These results suggest that spectral differences between FS and TO samples are a consequence of interference effects within the layers and thus an optical-geometrical artifact, as will be shown hereafter.

Even though in principle Eq. (1) is only valid for a single layer immersed in air, it clearly shows that the modulation pattern superimposed to the original PL spectrum from Sincs, i.e., separation between maxima-will strongly depend on the optical path followed by the light and thus on the incident and detection angles. Consequently, the PL spectra must change with these angles if interferences play any role at all. This is indeed the case, as the shape of the PL spectra from Si-ncs in fused silica substrates does not show dependence with incident or detection angles. On the contrary, we have observed dramatic differences in the spectral shape of Si-ncs in thermal oxide samples when varying the detection angle, as it is clearly illustrated in Fig. 5. On top of Fig. 5 we have plotted the PL spectra of 7\% Si excess FS sample for three different collection angles. The spectra are almost coincident and thus independent of detection angle. There is no modulation superimposed to the PL spectrum, which is a pure Gaussian profile. On the same figure we have represented a series of PL spectra as a function of detection angle for the corresponding TO sample. The shape spectacularly changes with the detection angle: the peak maximum shifts, the width changes, and for certain angles shoulders appear or even a splitting of the emission band (see Fig. 5). Then, we 


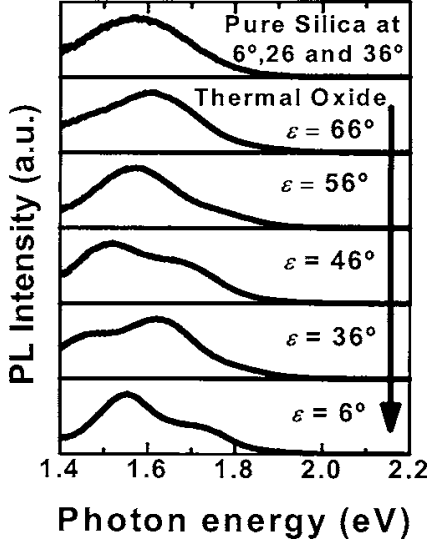

FIG. 5. Photoluminescence spectra from fused silica and thermal oxide samples with a $7 \%$ silicon excess for different detection angles.

can conclude that the origin of this phenomenon is the interferences produced within the $\mathrm{SiO}_{2}$ layer with thickness $d_{1}$ grown onto silicon and containing the sublayer with the embedded nanocrystals.

\section{SIMULATION OF OPTICAL MODULATION EFFECTS ON SI-NC PL SPECTRA}

We have been able to simulate the effect of interferences and then reproduce the PL spectra of Si-ncs in thermal oxide layers, by taking as a reference the spectra of Si-ncs in the corresponding silica samples, which show no interferences. These simulations provide a correction to the modulation superimposed to the spectra and thus allow obtaining of the actual spectra of $\mathrm{Si}$-nc without distortions.

We have represented in Fig. 6 a sketch of the structure of the TO samples, for which we have developed the calculations. Starting from the outer surface, it contains a 50-nmthick $\mathrm{SiO}_{2}$ cladding layer, a 300-nm-thick Si-nc-rich $\mathrm{SiO}_{2}$ layer, and a 1650 -nm-thick $\mathrm{SiO}_{2}$ buried layer on a $\mathrm{Si}$ substrate. This sequence well reproduces the structural characteristics of these samples, as obtained by the aforementioned characterization. The modeling of the PL emission has been performed in two steps: (i) calculating the continuous laser excitation and the profile of the related electromagnetic field inside the multilayered structure and (ii) the emission pattern, which depends on the excitation intensity and the location of the emitting Si-ncs.

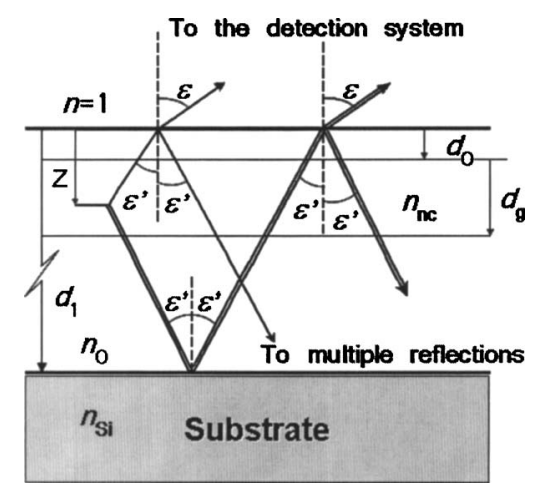

FIG. 6. Layer sequence used for the calculation of the resulting PL emission and the multiple reflections within the structure.

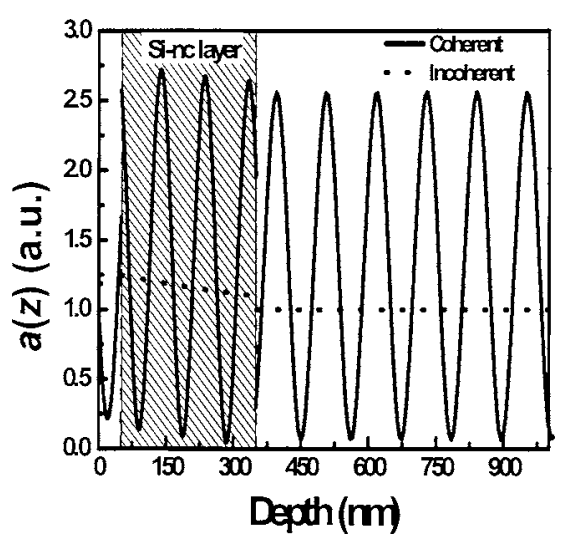

FIG. 7. Amplitude of the electric field of the excitation radiation $(325 \mathrm{~nm})$ along the oxide multilayer structure, as calculated by the simulator. The pattern has been normalized to the incident field.

In the first step, for the optical study of excitation in multilayered materials we have used the approach of Harbecke, ${ }^{20}$ which we have extensively developed and previously used in the study of vibrational spectra of multilayered systems. ${ }^{21}$ This method allows the calculation of the complex-amplitude reflection and transmission coefficients $(r$ and $t$ ) for a stack of planar absorbent layers. The transformation of fields across the interface between two layers and the propagation inside each layer are obtained from the wellknown matrix formalism developed by Abeles and Gittleman. ${ }^{22}$ One of the main features of this procedure is its ability to disregard the extremely narrow interference fringes of thick layers, then considering an incoherent propagation of light in the thick silica substrate. We have adapted the simulator to this particular work to obtain the amplitude of the excitation electric field as a function of depth $z$,

$$
a(z)=\frac{t_{a g}}{1-r_{g a} r_{g b} \varphi_{g}^{2}\left(d_{g}\right)}\left\{\varphi_{g}(z)+r_{g b} \varphi_{g}\left(d_{g}-z\right)\right\},
$$

where $d_{\mathrm{g}}$ is the thickness of layer $g$, and $\varphi$ stands for the propagation factor forward and backward within the active layer. The subindex of $r$ and $t$ indicates the transmission or reflection, respectively, of the whole stack from the top layer (subindex $a$ ) to the active layer $(g)$ and from the active layer to the buried $\mathrm{SiO}_{2}$ (subindex $b$ ). The angular dependence with $\theta$ is included in the coefficients $r$ and $t$.

Figure 7 illustrates the intensity profile of the $\mathrm{He}-\mathrm{Cd}$ excitation laser at $325 \mathrm{~nm}$ for the multilayered structure depicted in Fig. 6 corresponding to the 7\% Si excess TO sample. There is a sinusoidal pattern of interferences and the number of maxima can be predicted accurately with Eq. (1), in spite of its simplicity, if the absorption of the excitation radiation in the active layer is not strong (weakly absorbing medium). The number of nodes in the interference pattern increases when the excitation wavelength decreases or the refractive index increases, i.e., the same trend that Eq. (1) predicts. As one deals here with a flat profile of Si excess and the interference pattern shows several maxima and minima, the excitation energy density averaged along the whole Si-nc region is the same as the one obtained from a noncoherent source with the same incident power (Fig. 6). If, on the other hand, the Si-nc population would be confined into a very thin 
TABLE II. Refractive index and extinction coefficient of the Si nanocrystals layers in $\mathrm{SiO}_{2}$ for the various silicon excesses studied in this work.

\begin{tabular}{ccccc}
\hline \hline $\begin{array}{c}\text { Sample } \\
(\%)\end{array}$ & $n(325 \mathrm{~nm})$ & $k(325 \mathrm{~nm})$ & $n(700 \mathrm{~nm})$ & $k(700 \mathrm{~nm})$ \\
\hline 7 & 1.668 & 0.025 & 1.587 & 0.000 \\
9.5 & 1.702 & 0.056 & 1.620 & 0.002 \\
13 & 1.792 & 0.101 & 1.698 & 0.001 \\
\hline \hline
\end{tabular}

layer $\left(d_{g}<\lambda / 2 n_{0}\right)$ or would present a very irregular density profile, then the excitation intensity across it would show a dramatic dependence on the excitation wavelength, thickness, and refractive index of the active layer and cladding or substrate.

The simulator must be fed with the optical constants of all the layers at the excitation wavelength. The refractive index $n_{\mathrm{nc}}$ of the layer consisting of Si-ncs embedded in $\mathrm{SiO}_{2}$ has been obtained from spectroscopic ellipsometry and $m$ lines and modeled using the Bruggeman effective-medium approximation. $^{23}$ A detailed study concerning the complex refractive index of Si-ncs is not among the scopes of this paper and has been previously published by several authors. $^{24,25}$ With only the Si excess volume fraction as input, the complex refractive index of a $\mathrm{Si}-\mathrm{SiO}_{2}$ system can be easily calculated. Refractive index and extinction coefficient at the excitation wavelength $(325 \mathrm{~nm})$ and emitting wavelength $(700 \mathrm{~nm})$ are given in Table II for the three Si excesses studied here.

As far as the emission from the Si-nc ensemble is concerned, the light generated at each depth $z$ within the active layer is considered, giving rise to forward and backward propagating waves and their interference patterns. The results of the calculation provide a modulation function, which has to be superimposed to the actual Gaussian spectra of the $\mathrm{Si}$-ncs (that of the FS samples) to reproduce the experimental spectra for any given situation. This modulation function depends on the optical constants, oxide thickness, Si-nc region thickness, Si excess, incident and detection angles, and other geometrical parameters. For the calculation of the modulation functions, we have taken the complex refractive indices given in Table II for the composite layers in the 700-900 nm range, where the $\mathrm{Si}-\mathrm{ncs}$ emit. It is worth to point out that throughout this range the variation of the refractive index (less than $0.01 \%$ ) and the absolute value of the extinction coefficient (less than 0.002) of Si-ncs are negligible.

The basic procedure followed in this second step is described briefly hereafter. We have considered that a plane at depth $z$ emits in all directions (see Fig. 6). Only reflections between the air-oxide and oxide-silicon substrate interfaces are considered, because the contrast of the refractive index between $\mathrm{Si}$-nc-rich layer and pure $\mathrm{SiO}_{2}$ is low enough to consider that light suffers little deviation when crossing the interface among these two media. For a specific observation angle, two contributions to the final intensity are present, noted as the forward and the backward one, respectively. For each of them the geometric sum has to be made for all the succeeding reflections, taking into account the reflection and transmission coefficients and the phase-delay $\delta(z)$

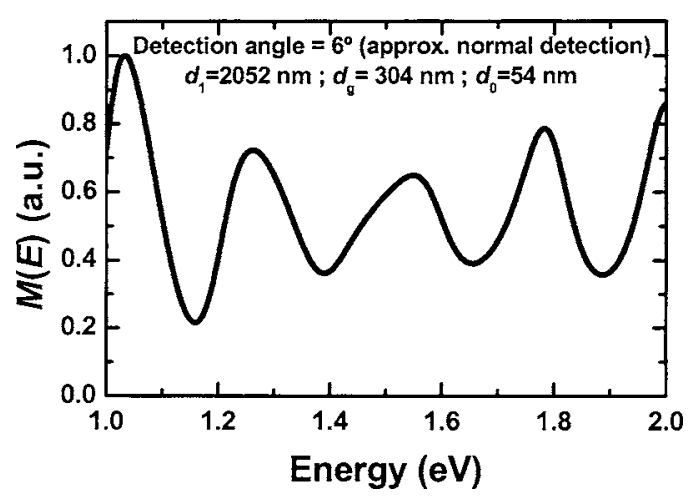

FIG. 8. Modulation function spectrum of the PL emission from Si nanocrystals embedded in thermal oxide, as calculated by the simulator.

$=4 \pi z n_{1} \cos \left(\varepsilon^{\prime}\right) / \lambda$, where $n_{1}$ is an average value of the refractive index over the whole $\mathrm{SiO}_{2}$ stack, $n_{1}\left[d_{g} n_{\mathrm{nc}}+\left(d_{1}\right.\right.$ $\left.\left.-d_{g}\right) n_{0}\right] / d_{1}$, while $\varepsilon^{\prime}$ is obtained from the Snell law, $n_{0} \sin \left(\varepsilon^{\prime}\right)=\sin (\varepsilon) .^{26}$ The complex coefficient is calculated and then the intensity factor, is integrated over the whole Si-nc emitting layer, considering that the emission of each $d z$ is incoherent with all the others. For nonpolarized light, the modulation function $M(E)$ is the average of both $s$ (parallel polarization) and $p$ (perpendicular polarization) waves. Then, the PL spectra can be written as the product of the modulation function and the Gaussian emission shape (centered at energy $E_{c}$ and with full width $w$ ), which has been independently determined in FS samples,

$$
I_{\mathrm{PL}}(E)=\frac{I}{w \sqrt{\pi / 2}} e^{-2\left(E-E_{C}\right)^{2} / w} M(E),
$$

and the modulation function is given by

$$
M(E)=\int_{d_{0}}^{d_{0}+d_{g}} \frac{a^{2}(z) t_{0}^{2}\left[1+r_{s}^{2}-2 r_{s} \sin \delta\left(d_{1}-z\right)\right]}{1+r_{0}^{2} r_{s}^{2}+2 r_{0} r_{s} \sin \delta\left(d_{1}\right)} d z,
$$

where $a(z)$ is the excitation profile amplitude calculated in Eq. (2), $t_{0}$ and $r_{0}$ are the transmission and reflection coefficients of the air-oxide interface, $r_{s}$ is the reflection coefficient of the oxide-substrate interface, and $\delta$ are the phase delays of the forward and backward contributions of the emission originated at depth $z$, which include the dependence on the collection angle $\varepsilon$.

\section{SIMULATION RESULTS AND DISCUSSION}

We have taken the emission shape of the samples synthesized in fused silica as the actual PL of the Si-ncs, free of any distortion. The Gaussian fitting following Eq. (2) gives $E_{c}=1.571 \pm 0.001 \mathrm{eV}$ and $w=0.248 \pm 0.001 \mathrm{eV}$ for the emission spectrum of FS sample with $7 \%$ of Si excess. This spectrum is the one already shown on top of Fig. 5. By calculating the modulation function for the corresponding $\mathrm{TO}$ sample and multiplying the result by the Gaussian fit, we have been able to reproduce with high accuracy its measured emission spectrum as a function of the detection angle, without making use of any free parameter (apart from an intensity normalization factor). The modulation function $M(E)$ is shown in Fig. 8 for a normal incidence/detection configura- 


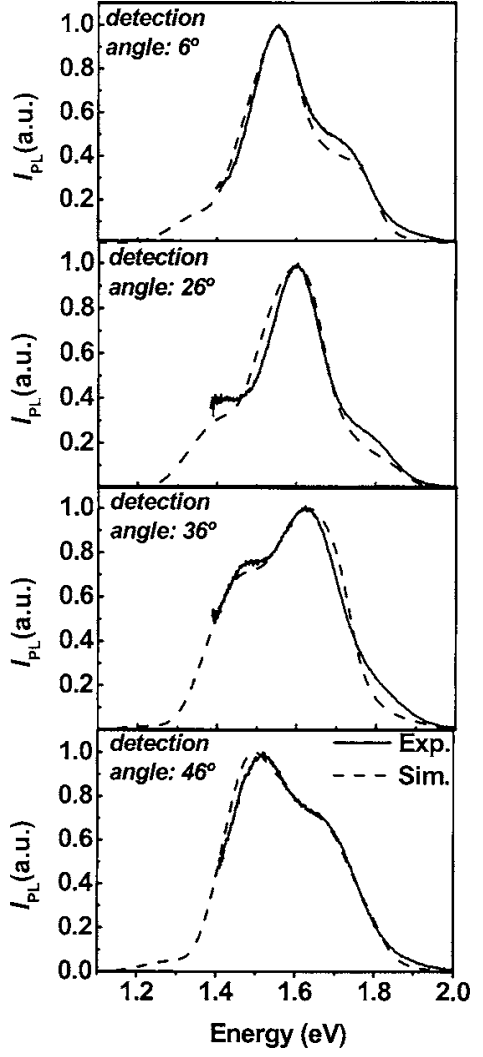

FIG. 9. Simulated and experimental PL spectra from a thermal oxide sample with the $7 \% \mathrm{Si}$ excess under different detection angles.

tion and the results of the simulation for this sample are shown in Fig. 9, superimposed to the experimental spectra for detection angles of $6^{\circ}, 26^{\circ}, 36^{\circ}$, and $46^{\circ}$.

Figure 9 demonstrates that it is possible to generate the spectral shape of the PL emission for any multilayer arrangement or experimental configuration by using the procedure stated above and starting with the undistorted spectrum of the Si-ncs. This demonstrates that many shifts, shoulders, and splits found in the emission spectra of Si-ncs can be only optical-geometrical artifacts. Ignoring these effects can give rise to an erroneous attribution of bands and average size determination of the Si-ncs. The explained procedure can be summarized as

$$
I_{\mathrm{PL}}^{\mathrm{expt}}(E)=M(E) I_{\mathrm{PL}}^{\mathrm{Si}-\mathrm{nc}}(E),
$$

where $I_{\mathrm{PL}}^{\mathrm{Si}-\mathrm{nc}}(E)$ indicates the undistorted PL emission from Si-ncs. The procedure can be easily reversed and then, once the modulation function $M(E)$ has been calculated, it is straightforward to obtain the "neat" spectrum from the experimental one regardless of the multilayer configuration or experimental arrangement.

Interferences and spectral distortions such as those shown above have been observed in other thermal oxide samples with different $\mathrm{Si}$ excesses, active layer thicknesses, and oxide thicknesses. Distortions appear at any detection angle, even at normal incidence, and this implies that one has to routinely correct the spectrum to get the true spectral shape of the Si-nc emission. Distortions would be significant and more detrimental when the thickness of the sample is such to give interferences with maxima separation of the

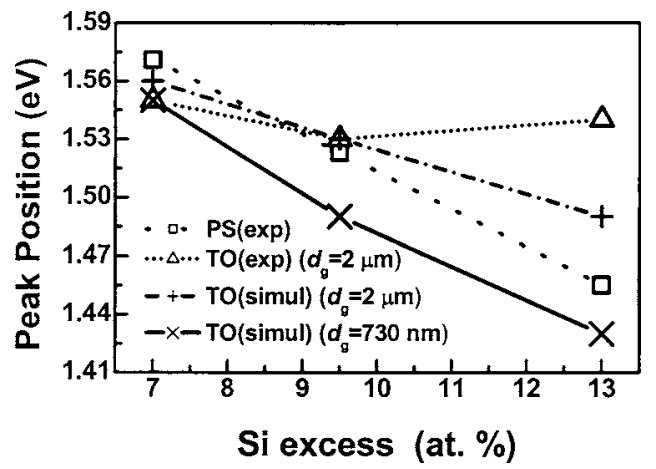

FIG. 10. Peak positions of the PL spectra from a thermal oxide sample resulting from simulations and from experiments. The peaks energies for the corresponding fused silica samples have been included.

same order of magnitude than the width of the emission band. Interferences and distortions would remain unnoticed for very thin oxides $(<0.3 \mu \mathrm{m})$ and for very thick ones $(>200 \mu \mathrm{m})$.

To demonstrate further the impact of distortions on the aforementioned PL band shifts, and the large errors which can arise by not taking into account these effects, we have used the simulator to predict the apparent, so-called "experimental," peak position of the PL emission from samples with different $\mathrm{Si}$ excesses, measured at normal incidence. Two different oxide thicknesses have been considered for simulation, $730 \mathrm{~nm}$ for a mild distortion and $2 \mu \mathrm{m}$, as before, for a relatively strong one. As shown in Fig. 10, there is a very good agreement between the predicted values and the ones found experimentally, in the case of the thick $\mathrm{SiO}_{2}$ layers. More interestingly, the simulation shows that just by changing some experimental conditions, in this case the thickness of the $\mathrm{SiO}_{2}$ layer underneath the region enriched with $\mathrm{Si}$ nanocrystals, dramatic changes in the variation of the peak position take place: From a PL band which looks rather insensitive to the size of the Si-nc population ( $2 \mu \mathrm{m}$ TO layer), up to an overestimation of the redshift (730-nm-thick TO layer) with respect to the true behavior, as measured in the silica samples.

\section{CONCLUSIONS}

We have demonstrated by experiments and simulations that optical-geometrical effects can be at the origin of pronounced shifts, spectral distortions, and even band splitting of the emission of $\mathrm{Si}$-ncs embedded in $\mathrm{SiO}_{2}$. Ignoring these effects can give rise to an erroneous attribution of bands and average size determination of the Si-ncs. We have also developed a procedure to calculate a modulation function which depends upon the multilayer configuration and the experimental arrangement and can be used to correct the experimental photoluminescence spectra and get the true peak position and emission shape of the Si-ncs. The simulations have been compared with experimental data and reproduce the spectral shifts and distortions that are observed when changing the thickness of the samples, the Si excess, and the detection angle. Only Si-ncs embedded in either thick 
samples, such as fused silica substrates or very thin oxides (less than $300 \mathrm{~nm}$ ), are free from spectral distortions due to interferences.

\section{ACKNOWLEDGMENTS}

This work has been financially supported by SINERGIA project (European Contract No. IST-2000-29650) and the Spanish project TIC2003-07464. The authors kindly acknowledge Dr. Caroline Bonafos of the CEMES/CNRS in Toulouse, France, and Dr. Jordi Arbiol of the University of Barcelona, Spain, for TEM analysis and valuable discussions.

${ }^{1}$ T. S. Iwayama, K. Fujita, S. Nakao, K. Sayito, F. Fujita, and N. Itoh, J. Appl. Phys. 75, 7779 (1994).

${ }^{2}$ L. Pavesi, L. Dal Negro, C. Mazzoleni, G. Franzò, and F. Priolo, Nature (London) 408, 440 (2000).

${ }^{3}$ M. H. Nayfeh, S. Rao, and N. Barry, Appl. Phys. Lett. 80, 121 (2002).

${ }^{4}$ G. Vijaya Prakash, M. Cazzanelli, Z. Gaburro, L. Pavesi, F. Iacona, G. Franzo, and F. Priolo, J. Appl. Phys. 91, 4607 (2002).

${ }^{5}$ T. S. Iwayama, N. Kurumado, D. E. Hole, and P. D. Townsend, J. Appl. Phys. 83, 6018 (1998).

${ }^{6}$ F. Iacona, C. Bongiorno, C. Spinella, S. Boninelli, and F. Priolo, J. Appl. Phys. 95, 3723 (2004).

B. Garrido, M. López, C. García, A. Pérez-Rodríguez, J. R. Morante, C. Bonafos, M. Carrada, and A. Claverie, J. Appl. Phys. 91, 2 (2002).
${ }^{8}$ H. J. Cheong, J. H. Kang, J. K. Kim, Y. Kim, J. Y. Yi, T. H. Chung, and H. J. Bark, Appl. Phys. Lett. 83, 2922 (2003).

${ }^{9}$ F. Iacona, G. Franzo, and C. Spinella, J. Appl. Phys. 87, 1295 (2000).

${ }^{10}$ M. L. Brongersma, P. G. Kik, A. Polman, K. S. Min, and H. A. Atwater, Appl. Phys. Lett. 76, 351 (2000).

${ }^{11}$ A. J. Kenyon, P. F. Trwoga, C. W. Pitt, and G. Rehm, J. Appl. Phys. 79, 9291 (1996).

${ }^{12}$ S. Botti, R. Coppola, F. Gourbilleau, and R. Rizk, J. Appl. Phys. 88, 3396 (2000).

${ }^{13}$ H. Yorikawa and S. Muramatsu, Appl. Phys. Lett. 71, 644 (1997).

${ }^{14}$ S. Guha, J. Appl. Phys. 84, 5210 (1998).

${ }^{15}$ S. Guha, S. B. Qadri, R. G. Musket, M. A. Wall, and T. Shimizu-Iwayama, J. Appl. Phys. 88, 3954 (2000).

${ }^{16}$ D. Nesheva, C. Raptis, A. Perakis, I. Bineva, Z. Aneva, Z. Levi, S. Alexandrova, and H. Hofmeister, J. Appl. Phys. 92, 4678 (2002).

${ }^{17}$ S. M. Orbons, M. G. Spooner, and R. G. Elliman, J. Appl. Phys. 96, 4650 (2004).

${ }^{18}$ R. G. Elliman, M. J. Lederer, and B. Luther-Davies, Appl. Phys. Lett. 80, 1325 (2002)

${ }^{19}$ P. Pellegrino et al., J. Appl. Phys. 97, 74312 (2005).

${ }^{20}$ B. Harbecke, Appl. Phys. B: Photophys. Laser Chem. 39, 165 (1986).

${ }^{21}$ J. A. Moreno, B. Garrido, J. Samitier, and J. R. Morante, J. Appl. Phys. 81, 1933 (1997).

${ }^{22}$ B. Abeles and J. I. Gittleman, Appl. Opt. 15, 10 (1976).

${ }^{23}$ D. A. G. Bruggeman, Ann. Phys. 24, 636 (1935).

${ }^{24}$ J. A. Moreno et al., J. Appl. Phys. 98, 13523 (2005).

${ }^{25}$ D. Amans, S. Callard, A. Gagnaire, J. Joseph, G. Ledoux, and F. Huiskens, J. Appl. Phys. 93, 4173 (2003).

${ }^{26}$ M. V. Klein and T. E. Furtak, Optics (Wiley, New York, 1986). 